\title{
Macho Detection by Pulsar Timing Observation
}

\author{
Kouji Ohnishi \\ Kansai Advanced Research Center, \\ Communications Research Laboratory, Kobe 651-24, Japan \\ Mizuhiko Hosokawa \\ Communications Research Laboratory, Koganei, Tokyo 184, Japan \\ Toshio Fukushima \\ National Astronomical Observatory, Mitaka, Tokyo 181, Japan \\ Mine Takeuti \\ Astronomical Institute, Tohoku University, Sendai 980-77, Japan
}

\section{Mass measurement by pulsar timing observation}

The magnitudes of some general relativistic effects depend on the mass of gravitating objects; therefore, these effects can be used for the stellar mass measurement. Several methods have been proposed; for example, the microlensing effect (Paczyński 1986), and the parallactic variation of gravitational deflection of the light from the source (Hosokawa et al. 1993).

Recently some candidates for MACHOs - a kind of dark matter candidates in the Galactic halo - have been discovered by their microlensing effect, and their masses were estimated to be of the order of $0.1 M_{\odot}$, under many assumptions. Actual measurement of MACHO masses are important in specifying what kind of object the MACHO is.

We proposed a new method: the variation of the gravitational time delay of pulses from a pulsar caused by the motion of foreground stars relative to the pulsar in the pulsar timing observation (Ohnishi et al. 1995). Consider that the observer, the foreground star and the pulsar are well aligned. In this situation, the gravitational time delay due to the proper motion of the star relative to the pulsar is expressed as

$$
\Delta t(t)=\text { constant }-2 \frac{G M}{c^{3}} \ln \left|1+\left(\frac{t-t_{0}}{t_{d}}\right)^{2}\right| .
$$

where $G$ is the gravitational constant, $M$ is the mass of the foreground star, $c$ is the speed of light, $t_{0}$ is the epoch of the closest approach and $t_{\mathrm{d}}$ is the ratio of the minimum separation angle to the relative proper motion $\mu$ that characterizes the time scale of this effect.

This gravitational delay as a function of time has two inflection points and will make a positive hump in the residuals of the time of arrival (TOA) of pulses 
after fitting to the existing models of the TOA analysis. When such a property is observed in the residuals of the TOA, the masses of the stars can be determined from the TOA data analysis by introducing three new parameters $M, t_{0}$, and $t_{d}$. Note that if the observation period $T$ does not cover both of the inflection points of $\Delta t(t)$, this effect will be observed as the false cubic trend in the timing residual.

\section{Detection probability}

This method can be applied to the detection of MACHOs. The probability of MACHOs detection $\Gamma$ is expressed as

$$
\Gamma=\int_{0}^{D_{\mathrm{P}}} \mathrm{d} D_{\mathrm{S}} k\left(\mu T D_{\mathrm{S}}\right)^{2} n\left(l, b, D_{\mathrm{S}}\right)
$$

where $D_{\mathrm{P}}$ and $D_{\mathrm{S}}$ are the distance of the pulsar and MACHO, respectively, $n$ is the number density of MACHOs, $l$ and $b$ are the galactic longitude and latitude, respectively, and $k$ is the constant that depends on timing accuracy. Here we assume that the distribution of MACHOs is isothermal, their masses are $0.1 M_{\odot}$ and their velocities are $200 \mathrm{~km} / \mathrm{s}$, then the detection probability during 10 years' observation with the timing accuracy of $200 \mathrm{~ns}$ is several thousandth per each pulsars.

Table I Detection probabilities for some millisecond pulsars

\begin{tabular}{lcrrlcc} 
Pulsar & $P(m s)$ & $l$ & $b$ & $D_{\mathrm{P}}(k p c)$ & $V_{\mathrm{P}}(\mathrm{km} / \mathrm{s})$ & $\Gamma$ \\
\hline PSR J0437-4715 & 5.75 & 253.4 & -42.0 & 0.15 & 96 & $1 \times 10^{-4}$ \\
PSR J1713+0747 & 4.57 & 29 & 25 & 1.1 & 34 & $1 \times 10^{-3}$ \\
PSR B1937+21 & 1.56 & 57.5 & -0.3 & 3.6 & 10 & $4 \times 10^{-3}$ \\
\hline
\end{tabular}

These probabilities might be small, however, they are proportional to the square of the observation period. Further, many new millisecond pulsars will be found as the observation sensitivity improves. In the future, a longer observation period and a more precise and sensible observation will make the detection of this effect easier.

\section{References}

Hosokawa, M., Ohnishi, K., Fukushima, T. \& Takeuti, M. 1993, A\&A, 278, L27 Ohnishi, K., Hosokawa, M., Fukushima, T. \& Takeuti, M. 1995, ApJ, 448, 271 Paczyński, B. 1986, ApJ, 304, 1 